\title{
Timing, severity of deficits, and clinical improvement after surgery for spinal dural arteriovenous fistulas
}

\author{
Michael M. Safaee, MD, ${ }^{1}$ Aaron J. Clark, MD, PhD, ${ }^{1}$ Jan-Karl Burkhardt, MD, ${ }^{1}$ \\ Ethan A. Winkler, MD, PhD, ${ }^{1}$ and Michael T. Lawton, MD² \\ 1Department of Neurological Surgery, University of California, San Francisco, California; and 'Department of Neurological \\ Surgery, Barrow Neurological Institute, Phoenix, Arizona
}

\begin{abstract}
OBJECTIVE Spinal dural arteriovenous fistulas (dAVFs) are rare vascular abnormalities caused by arteriovenous shunting. They often form at the dural root sleeve between a radicular feeding artery and draining medullary vein causing venous congestion and edema, decreased perfusion, and ischemia of the spinal cord. Treatment consists of either surgical ligation of the draining vein or selective embolization via an endovascular approach. There is a paucity of data on which modality provides more durable and effective outcomes.
\end{abstract}

METHODS The authors performed a retrospective review of a prospectively maintained database by the senior author to assess clinical outcomes in patients undergoing surgical treatment of spinal dAVFs. Preoperative and postoperative motor and Aminoff-Logue Scale (ALS) scores were collected.

RESULTS A total of 41 patients with 44 spinal dAVFs were identified, with a mean patient age of 64 years. The mean symptom duration was 14 months, with weakness ( $82 \%)$, urinary symptoms (47\%), and sensory symptoms $(29 \%)$ at presentation. The fistula locations were as follows: 30 thoracic, 9 lumbar, 3 sacral, and 2 cervical. Five patients had normal motor and ALS scores at presentation. Among the remaining 36 patients with motor deficits or abnormal gait and micturition at presentation, $78 \%$ experienced an improvement while the remaining $22 \%$ continued to be stable. There was a trend toward improved outcomes in patients with shorter symptom duration; mean symptom duration among patients with clinical improvement was 13 months compared with 22 months among those without improvement. Additionally, rates of improvement were higher for lower thoracic and lumbosacral dAVFs (85\% and $83 \%$ ) compared with those in the upper thoracic spine (57\%). No patient developed recurrent fistulas or worsening neurological deficits.

CONCLUSIONS Surgery is associated with excellent outcomes in the treatment of spinal dAVFs. Early diagnosis and treatment are critical, with a trend toward improved outcomes. No patient in this study had fistula recurrence or worsening of symptoms. Among patients with abnormal motor or ALS scores, $78 \%$ improved after surgery. Therapeutic embolization is an option for some lesions, but for cases with unfavorable anatomy where embolization is not feasible, surgery is a safe option associated with high success.

https://thejns.org/doi/abs/10.3171/2017.11.SPINE17988

KEYWORDS spine; dural arteriovenous fistula; surgical outcomes; vascular disorders

$\mathrm{S}$ PINAL dural arteriovenous fistulas (dAVFs) are caused by an arteriovenous shunt between one or more branches of a dural artery and the medullary venous system. They are often from dural arteries supplying the dural root sleeve and adjacent spinal dura and a draining medullary vein that drains the coronal venous plexus. ${ }^{4,13}$ This leads to retrograde filling of the venous plexus with subsequent congestion, dilation, and venous hypertension within the spinal cord, resulting in edema, reduced perfusion, and ischemia of the cord causing a slow and progressive myelopathy. ${ }^{1,9}$ Patients generally present with chronic and progressive gait instability and motor and sensory symptoms in the lower extremities. ${ }^{10}$ There is often a discrepancy between the location of a dAVF and symptomatology, suggesting that the site of venous drainage determines clinical presentation. ${ }^{8,11}$ Due to the relative

ABBREVIATIONS ALS = Aminoff-Logue Scale; $d A V F=$ dural arteriovenous fistula; ICG = indocyanine green; MRC = Medical Research Council.

SUBMITTED September 4, 2017. ACCEPTED November 16, 2017.

INCLUDE WHEN CITING Published online April 20, 2018; DOI: 10.3171/2017.11.SPINE17988. 
rarity of these lesions and chronicity of symptoms, the appropriate diagnostic workup is often delayed and the time from presentation to diagnosis is often long.

Traditional therapy for these lesions consists of surgery for ligation of the draining vein at the fistula point. Newer endovascular therapies exist with selective embolization; however, this is not always possible based on vascular anatomy. The appropriateness of each approach and durability of endovascular versus open surgery remain points of debate with a paucity of high-level data. In this study, we present our 20-year experience in the management of these lesions with specific insights on timing of surgery and neurological outcomes.

\section{Methods}

\section{Patient Demographics and Outcomes}

Patients were identified through a retrospective review of our neurovascular surgery database that includes all patients operated on by the senior author (M.T.L.) since 1997. All activities were approved by the University of California, San Francisco Committee on Human Research (our IRB). All adult patients with a diagnosis of spinal dAVF who underwent surgical treatment were included. All surgeries were performed at a single institution by the senior author (M.T.L.). Symptom duration was defined as the time from symptom onset to surgery. T2 signal change and the level of shunting in the spinal cord were determined by review of preoperative MRI and angiography, respectively; both were independently confirmed by a neuroradiologist. Fistula locations were classified as cervical, thoracic, lumbar, or sacral.

Motor strength at presentation was reported using the standard Medical Research Council (MRC) motor grading system for the weakest limb as follows: $0=$ complete paralysis, $1=$ minimal contraction, $2=$ active movement with gravity eliminated, $3=$ active movement against gravity, $4-=$ active movement against gravity and minimal resistance, $4=$ active movement against gravity and mild resistance, $4+=$ active movement against gravity and moderate resistance, and $5=$ normal strength. The Aminoff-Logue Scale (ALS) measures gait (range 0-5) and micturition (range $0-3$ ) as follows: gait score $0=$ normal; 1 $=$ leg weakness, abnormal gait or stance, but no restriction of activity; 2 = restricted activity; 3 = requiring 1 stick for walking; $4=$ requiring 2 sticks, crutches, or walker; and 5 $=$ confined to wheelchair; micturition score $0=$ normal; 1 $=$ hesitancy, urgency, or frequency; $2=$ occasional urinary incontinence or retention; and $3=$ total incontinence or retention. Outcomes were classified as stable, improved, or worse based on changes in the MRC and ALS scores. If any score was worse postoperatively, the overall outcome was classified as "worse," while if at least one symptom improved, the outcome was classified as "improved."

\section{Surgical Technique and Perioperative Care}

All lesions were approached posteriorly with the patient prone with either a Mayfield head clamp for cervical or upper thoracic lesions or a Wilson frame for lower thoracic and lumbar lesions. Intraoperative neuromonitoring with somatosensory evoked potentials and motor evoked potentials was used for all cases. Steroids were administered preoperatively and continued for 48 hours postoperatively. Intraoperative systolic blood pressure was maintained at less than $140 \mathrm{~mm} \mathrm{Hg}$ and liberalized to less than $160 \mathrm{~mm} \mathrm{Hg}$ postoperatively.

After confirming the appropriate spinal level with fluoroscopy, 1- or 2-level laminectomies were performed based on the fistula size and drainage pattern. The microscope was brought into the operative field and the dura and arachnoid over the spinal cord or cauda equina were opened using standard microsurgical techniques. For cervical and thoracic dAVFs special care was taken to avoid mobilizing the spinal cord, whereas for lumbar or sacral dAVFs the nerve roots were carefully displaced to identify the arterialized draining vein. In general, the arterialized draining vein was identified laterally along the segmental nerve roots. The arterialized draining vein is typically dilated and thickened and shows whitening of its walls at the fistula site. Indocyanine green (ICG) video angiography was used later in the series to confirm the draining vein and the site of arteriovenous shunting. The vein was clip occluded and then divided, and ICG angiography confirmed complete obliteration of the fistula. There is no need to occlude or coagulate arteries in the dura, as they thrombose once their outflow is occluded. Representative angiography and intraoperative images from one patient are shown in Fig. 1.

The dura is closed in a watertight fashion and patients are transferred to the intensive care unit for overnight observation. Patients were maintained on bed rest in the supine position for 24 hours to promote healing of the durotomy, then mobilized with physical therapy. All patients underwent postoperative angiography to confirm fistula obliteration.

\section{Statistical Analysis}

Univariate analysis of continuous variables was performed using the Student t-test and categorical variables were compared by the chi-square test. Statistical significance was defined as $p<0.05$. All analyses were performed with SPSS (version 24, IBM Corp.).

\section{Results}

\section{Patient Characteristics}

A total of 41 patients with 44 spinal dAVFs were identified with a mean patient age of 64 years (range 35-85 years). This study population included 30 men and 11 women with an average symptom duration of 14 months. The presenting symptoms were lower-extremity weakness $(81 \%)$, urinary symptoms (49\%), and sensory symptoms (34\%). One patient had an incidentally found cervical dAVF that was detected during an angiogram after subarachnoid hemorrhage from a ruptured intracranial aneurysm. Four additional patients had vague sensory symptoms with normal motor and gait/micturition function. Preoperative MRC scores in the weakest limb were as follows: $0 / 5$ in 3 patients (7\%), $1 / 5$ in 7 patients (17\%), $2 / 5$ in 4 patients $(10 \%), 3 / 5$ in 3 patients (7\%), $4-/ 5$ in 3 patients (7\%), $4 / 5$ in 3 patients (7\%), $4+/ 5$ in 8 patients (20\%), and $5 / 5$ in 10 patients (25\%). Fourteen patients (34\%) had 

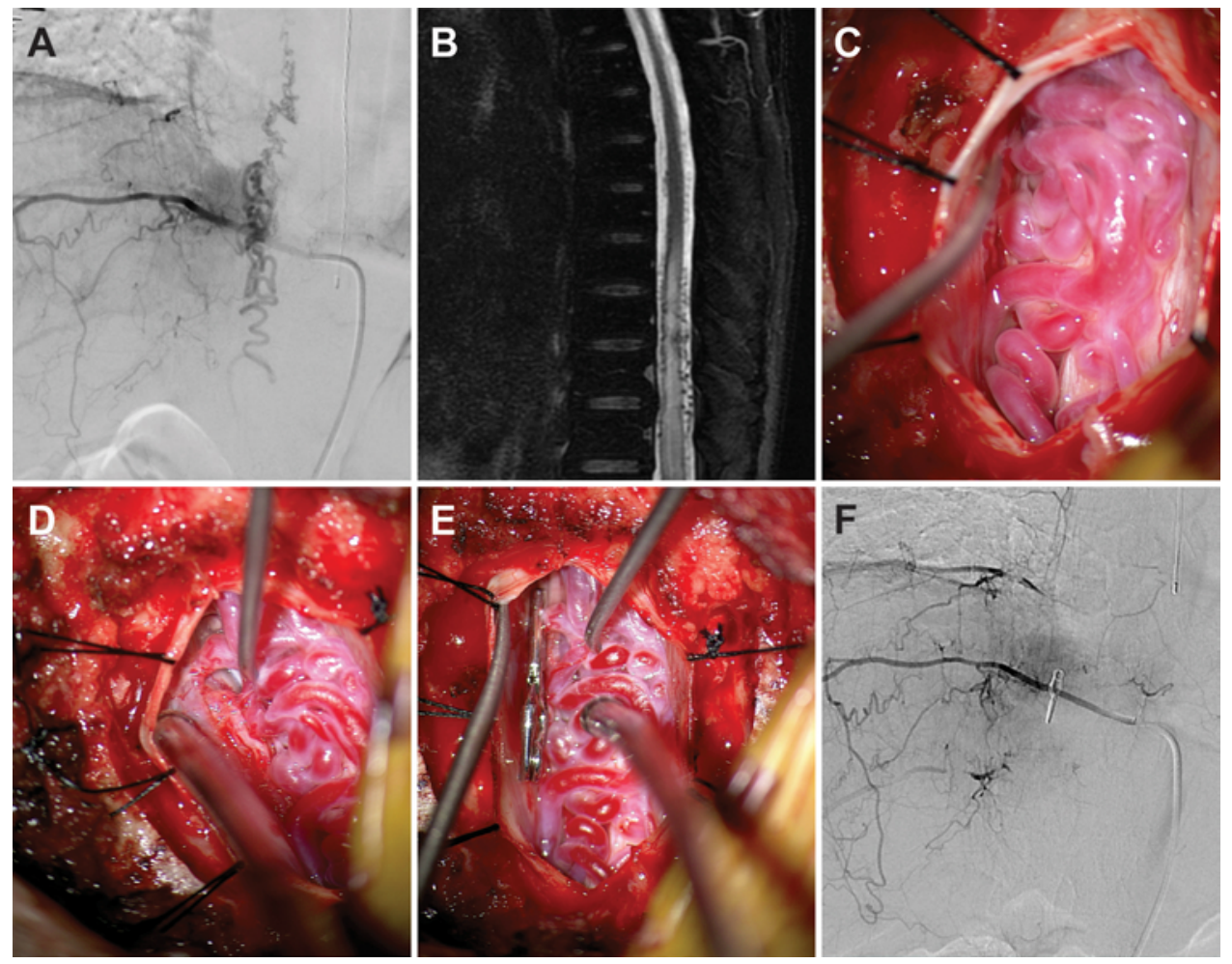

FIG. 1. Representative angiography and surgical results following clip ligation of a spinal dAVF. A 62-year-old woman presented with 12 months of progressive lower-extremity weakness, gait instability, and occasional urinary retention. A: Anterior-posterior projection of preoperative angiogram showing T-10 dAVF supplied by the radicular meningeal branch. Injection, right T-10 intercostal artery. B: Midsagittal T2-weighted MRI demonstrated dorsal flow voids and intramedullary T2 hyperintensity consistent with venous hypertension and edema in the mid- to low-thoracic spinal cord. C-E: Intraoperative photography demonstrating linear dural opening after 2-level laminectomy. Once the lesion is visualized, careful microdissection isolates the arterial fistula, which enters the thecal sac through the nerve root sheath ( $D$ and $E)$. The fistulous connection is clipped with a single surgical clip (E). To ensure complete disruption, the fistula is then cut distal to the surgical clip. F: Anterior-posterior projection of postoperative angiogram showing complete obliteration. Injection, right T-10 intercostal artery. Figure is available in color online only.

less than antigravity strength at presentation. Preoperative ALS scores and patient demographics are summarized in Table 1 . Among 41 patients, only 2 had multiple spinal dAVFs. Locations of the dAVFs were as follows: 2 cervical, 30 thoracic, 9 lumbar, 3 sacral. In $34(83 \%)$ of 41 patients the preoperative MRI showed a T2 signal in the spinal cord. Interestingly, 32 (89\%) of 36 patients with preoperative motor or gait/micturition symptoms had preoperative T2 cord signal abnormalities compared with just $2(40 \%)$ of 5 patients without symptoms $(\mathrm{p}=0.006)$. Embolization was attempted in 6 patients $(15 \%)$, but this resulted in only partial occlusion in 5 cases and recurrence in 1 case.

\section{Surgical Outcomes}

All lesions were approached using posterior laminectomy. In each case, a complete surgical cure was obtained and confirmed by postoperative angiography. The average length of follow-up was 13 months (range 1-76 months). Among the 31 patients with motor deficits, 24 (77\%) improved; among the 35 patients with abnormal ALS scores, 25 (71\%) improved. Among all 36 patients with a motor and/or gait/micturition deficit, 28 (78\%) had an improvement in one or more modalities. In patients with preop- erative motor deficits, the presence of antigravity strength $(\mathrm{MRC} \geq 3)$ was not associated with a better rate of improvement $(79 \%$ vs $76 \%, \mathrm{p}=0.889)$. No patient had a worse MRC or ALS score after surgery. Postoperative angiography confirmed successful obliteration of all but one fistula; this patient required a return to the operating room for treatment of a residual fistula with a successful outcome. Postoperative outcomes are summarized in Table 2.

\section{Symptom Duration}

Among patients with abnormal MRC or ALS scores at presentation, there was a trend toward improved outcomes with shorter symptom duration. Among the 28 patients with an improvement in either modality, the mean symptom duration was 13 months compared with 22 months among those with no improvement $(p=0.252)$. When assessing motor deficits alone, the mean symptom duration among patients with improvement after surgery was 14 months compared with 24 months among those without improvement $(p=0.244)$. When assessing gait or micturition deficits alone, the mean symptom duration was 13 months among those with improvement after surgery compared with 19 months in those without improvement $(\mathrm{p}=0.385)$. 
TABLE 1. Preoperative demographics in 41 patients with spinal dAVFs

\begin{tabular}{|c|c|}
\hline Variable & Value \\
\hline \multicolumn{2}{|l|}{ Age (yrs) } \\
\hline Mean (range) & $64(35-85)$ \\
\hline \multicolumn{2}{|l|}{ Sex, no. $(\%)$} \\
\hline Female & $11(27)$ \\
\hline Male & $30(73)$ \\
\hline \multicolumn{2}{|l|}{ Symptom duration (mos) } \\
\hline Mean (range) & $14(0-78)$ \\
\hline \multicolumn{2}{|l|}{ Presenting symptoms, no. (\%) } \\
\hline Weakness & $33(81)$ \\
\hline Urinary symptoms & $20(49)$ \\
\hline Sensory symptoms & $14(34)$ \\
\hline \multicolumn{2}{|l|}{ Preop MRC score, no. $(\%)^{*}$} \\
\hline 5 & $10(24)$ \\
\hline $4+$ & $8(20)$ \\
\hline 4 & $3(7)$ \\
\hline $4-$ & $3(7)$ \\
\hline 3 & $3(7)$ \\
\hline 2 & $4(10)$ \\
\hline 1 & $7(17)$ \\
\hline 0 & $3(7)$ \\
\hline \multicolumn{2}{|l|}{ Preop ALS score, no. (\%) } \\
\hline 0 & $6(15)$ \\
\hline 1 & $4(10)$ \\
\hline 2 & $2(5)$ \\
\hline 3 & $6(15)$ \\
\hline 4 & $2(5)$ \\
\hline 5 & $4(10)$ \\
\hline 6 & $5(12)$ \\
\hline 7 & $9(22)$ \\
\hline 8 & $3(7)$ \\
\hline \multicolumn{2}{|l|}{ Location of dAVF, no. (\%)† } \\
\hline Cervical & $2(5)$ \\
\hline Thoracic & $30(68)$ \\
\hline Lumbar & $9(20)$ \\
\hline Sacral & $3(7)$ \\
\hline \multicolumn{2}{|l|}{ No. of dAVFs, no. (\%) } \\
\hline 1 & $39(95)$ \\
\hline 2 & $1(2.4)$ \\
\hline 3 & $1(2.4)$ \\
\hline Preop T2 signal, no. (\%) & $34(83)$ \\
\hline Preop embolization, no. (\%) & $6(15)$ \\
\hline
\end{tabular}

* Strength in weakest limb.

$\dagger$ Among 44 lesions in 41 patients.

\section{Fistula Location}

With respect to fistula location, we assessed all 44 fistulas independently. Among 39 symptomatic lesions, there was a trend toward improved outcomes among lesions lower in the spinal cord. Rates of overall improvement were $57 \%$ for lesions in the upper thoracic cord (defined as T1-6), 85\% for lesions in the lower thoracic cord (defined as T7-12), and 83\% for lumbosacral lesions ( $\mathrm{p}=$ 0.269). With respect to motor deficits, rates of improvement for upper thoracic lesions were $50 \%$ compared with
TABLE 2. Clinical outcomes in 36 symptomatic patients undergoing surgical treatment for spinal dAVFs

\begin{tabular}{cc}
\hline \multicolumn{1}{c}{ Variable } & Value $(\%)$ \\
\hline Overall outcomes ${ }^{*}$ & \\
Improved & $28(78)$ \\
Same & $8(22)$ \\
Worse & $0(0)$ \\
\hline Motor improvement† & $24(77)$ \\
\hline ALS improvement $¥$ & $25(71)$ \\
\hline Postop MRC score§ & \\
5 & $14(39)$ \\
$4+$ & $6(17)$ \\
4 & $4(11)$ \\
$4-$ & $4(11)$ \\
3 & $3(8)$ \\
2 & $2(6)$ \\
1 & $2(6)$ \\
0 & $1(3)$ \\
\hline Postop ALS score & \\
0 & $9(25)$ \\
1 & $4(11)$ \\
2 & $0(0)$ \\
3 & $4(11)$ \\
4 & $5(14)$ \\
5 & $3(8)$ \\
6 & $7(19)$ \\
7 & $2(6)$ \\
8 & $2(6)$ \\
\hline
\end{tabular}

* Among 36 patients with abnormal MRC or ALS scores preoperatively.

$\dagger$ Among 31 patients with preoperative motor deficits.

$\ddagger$ Among 35 patients with preoperative urinary or gait symptoms.

$\S$ Strength in weakest limb.

$89 \%$ for lower thoracic and $80 \%$ for lumbosacral lesions $(\mathrm{p}=0.125)$. Regarding gait/micturition deficits, rates of ALS improvement were $67 \%$ for upper thoracic lesions and $75 \%$ each for both lower thoracic and lumbosacral lesions $(\mathrm{p}=0.913)$. These results are summarized in Table 3. Individual patient outcomes are summarized in Table 4.

\section{Preoperative T2 Cord Signal Change}

We assessed whether the presence of $\mathrm{T} 2$ cord signal change was associated with rates of neurological improvement. Among 31 patients with preoperative motor deficits, rates of improvement after surgery were $78 \%$ (21 of 27) among patients with cord signal change compared with $75 \%$ (3 of 4) among patients without cord signal change $(\mathrm{p}=0.901)$. Among 35 patients with gait or urinary symptoms, rates of ALS score improvement were $75 \%$ (24 of 32) among those with abnormal cord signal compared with $33 \%$ (1 of 3 ) in those without cord signal changes ( $\mathrm{p}=$ $0.127)$. Among all 36 symptomatic patients, the rate of any neurological improvement in patients with preoperative cord signal abnormalities was $78 \%$ (25 of 32) compared with $75 \%$ ( 3 of 4 ) in those without ( $\mathrm{p}=0.887)$.

\section{Preoperative Embolization}

We assessed outcomes of preoperative embolization as 
TABLE 3. Outcome by dAVF location

\begin{tabular}{ccc}
\hline Outcome & No. of dAVFs $(\%)$ & p Value \\
\hline Overall improvement* & & 0.269 \\
Upper thoracic & $4 / 7(57)$ & \\
Lower thoracic & $17 / 20(85)$ & \\
Lumbosacral & $10 / 12(83)$ & 0.125 \\
\hline Motor improvement† & & \\
Upper thoracic & $3 / 6(50)$ & \\
Lower thoracic & $16 / 18(89)$ & 0.913 \\
Lumbosacral & $8 / 10(80)$ & \\
ALS improvement & & \\
Upper thoracic & $4 / 6(67)$ & \\
Lower thoracic & $15 / 20(75)$ & \\
Lumbosacral & $9 / 12(75)$ & \\
\hline
\end{tabular}

Upper thoracic $=\mathrm{T} 1-6$; lower thoracic $=\mathrm{T} 7-12$.

* Among all 39 symptomatic dAVFs.

$\dagger$ Among 34 dAVFs with associated preoperative motor deficits.

$\ddagger$ Among 38 dAVFs with associated preoperative gait or urinary abnormalities.

an adjunct to surgical ligation. In these 6 cases, 5 were partial embolizations due to vascular anatomy that precluded complete occlusion and 1 was a recurrence after previous embolization. Among 31 patients with preoperative motor deficits, 4 (67\%) of 6 who underwent preoperative partial embolization had an improvement in MRC score compared with $20(80 \%)$ of 25 who underwent surgery alone $(\mathrm{p}=0.483)$. Among 35 patients with gait or urinary symptoms, 4 (67\%) of 6 who underwent preoperative partial embolization showed an improvement in ALS score compared with $21(72 \%)$ of 29 in those who underwent surgery alone $(\mathrm{p}=0.777)$. Among all 36 symptomatic patients, $4(67 \%)$ of 6 of those who underwent embolization demonstrated an improvement compared with $24(80 \%)$ of 30 without embolization $(\mathrm{p}=0.473)$.

\section{Discussion}

Without treatment, spinal dAVFs will invariably cause worsening symptoms and progressive neurological deficit. The significant challenge in treating patients with spinal dAVFs is making the appropriate diagnosis and doing so in a timely manner. Given the sometimes indolent and variable clinical presentation, it is not uncommon for patients to present many months after their initial symptoms. In this series, 18 (44\%) of 41 patients presented more than 12 months after symptom onset. There are no convincing data that early diagnosis and treatment are associated with improved outcomes, despite conventional wisdom. In fact, Cenzato et al. found that patients with more acute presentations had lower rates of neurological improvement compared with those with more chronic symptoms (70\% vs $81 \%) .{ }^{3}$ Furthermore, no patient in that study fared worse after surgery. Our data, however, suggest a trend toward improved outcome in patients with early treatment; mean symptom duration among symptomatic patients with neurological improvement was 13 months compared with 22 months in those without improvement in either MRC or ALS scores. Although this did not reach statistical significance, there is compelling biological rationale to suggest
TABLE 4. Individual patient outcomes

\begin{tabular}{|c|c|c|c|c|c|c|}
\hline \multirow{2}{*}{$\begin{array}{l}\text { Age } \\
\text { (yrs), } \\
\text { Sex }\end{array}$} & \multirow{2}{*}{$\begin{array}{c}\text { Duration of } \\
\text { Symptoms } \\
\text { (mos) }\end{array}$} & \multirow{2}{*}{$\begin{array}{c}\text { dAVF } \\
\text { Location }\end{array}$} & \multicolumn{2}{|c|}{ MRC Score } & \multicolumn{2}{|c|}{ ALS Score } \\
\hline & & & Preop & Postop & Preop & Postop \\
\hline $70, \mathrm{M}$ & 12 & $\mathrm{~T}-11$ & 1 & 2 & 5 & 4 \\
\hline $69, \mathrm{M}$ & 48 & $\mathrm{~T}-11$ & 4 & $4+$ & 2 & 0 \\
\hline $80, \mathrm{~F}$ & 78 & T-5 & 0 & 0 & 8 & 8 \\
\hline $83, \mathrm{~F}$ & 30 & $\mathrm{~T}-7$ & $4+$ & 5 & 4 & 3 \\
\hline $60, \mathrm{~F}$ & 1 & L-1 & 1 & 2 & 7 & 7 \\
\hline $60, \mathrm{M}$ & 3 & $T-9 \& T-12$ & 0 & $4+$ & 6 & 4 \\
\hline $62, \mathrm{M}$ & 5 & T-4 & 4 & $4+$ & 7 & 3 \\
\hline $67, \mathrm{M}$ & 2 & L-3 & 3 & 4- & 5 & 4 \\
\hline $50, \mathrm{M}$ & 1 & $\mathrm{~T}-7$ & $4+$ & 5 & 3 & 4 \\
\hline $56, \mathrm{~F}$ & 18 & $\mathrm{~T}-11, \mathrm{~L}-1, \mathrm{~L}$ L-2 & $4+$ & 5 & 3 & 0 \\
\hline $70, \mathrm{~F}$ & 24 & $\mathrm{~T}-8$ & 4 & $4+$ & 6 & 0 \\
\hline $56, \mathrm{~F}$ & 23 & L-4 & 2 & 3 & 7 & 6 \\
\hline $69, \mathrm{M}$ & 12 & $\mathrm{~T}-6$ & 3 & 3 & 6 & 6 \\
\hline $69, \mathrm{M}$ & 4 & $\mathrm{~T}-7$ & 3 & 5 & 3 & 6 \\
\hline $53, \mathrm{M}$ & 6 & T-9 & 5 & 5 & 0 & 1 \\
\hline $49, \mathrm{M}$ & 8 & $\mathrm{~T}-11$ & 5 & 5 & 1 & 0 \\
\hline $62, \mathrm{M}$ & 2 & $\mathrm{~T}-7$ & 5 & 5 & 1 & 1 \\
\hline $50, \mathrm{M}$ & 8 & L-1 & 2 & 4 & 7 & 0 \\
\hline $35, \mathrm{M}$ & 7 & $\mathrm{~T}-10$ & 2 & 5 & 4 & 6 \\
\hline $48, \mathrm{M}$ & 0 & C-2 & 5 & 5 & 0 & 3 \\
\hline $73, \mathrm{M}$ & 10 & L-3 & 1 & 1 & 5 & 0 \\
\hline $85, \mathrm{~F}$ & 6 & L-3 & 4- & 4- & 7 & 5 \\
\hline $56, \mathrm{~F}$ & 12 & C-3 & 5 & 5 & 0 & 7 \\
\hline $65, M$ & 5 & S-1 & 1 & 4- & 6 & 5 \\
\hline $67, \mathrm{M}$ & 1 & T-6 & 0 & 4 & 7 & 6 \\
\hline $79, \mathrm{~F}$ & 5 & L-12 & 1 & 4 & 6 & 4 \\
\hline $64, \mathrm{M}$ & 60 & $\mathrm{~T}-7$ & $4+$ & 5 & 3 & 0 \\
\hline $48, M$ & 9 & T-6 & 5 & 5 & 2 & 0 \\
\hline $50, \mathrm{M}$ & 36 & $\mathrm{~T}-1$ & $4+$ & 5 & 0 & 0 \\
\hline $63, \mathrm{M}$ & 11 & $\mathrm{~T}-7$ & 5 & 5 & 0 & 0 \\
\hline $79, \mathrm{M}$ & 1 & $\mathrm{~T}-10$ & $4+$ & 5 & 1 & 0 \\
\hline $63, \mathrm{M}$ & 24 & $\mathrm{~T}-11$ & 1 & 3 & 7 & 6 \\
\hline $68, \mathrm{M}$ & 24 & L-1 & 5 & 5 & 7 & 4 \\
\hline $84, \mathrm{~F}$ & 20 & T-9 & $4+$ & 5 & 7 & 3 \\
\hline $62, F$ & 12 & $\mathrm{~T}-10$ & 4- & $4-$ & 5 & 5 \\
\hline $72, \mathrm{M}$ & 1 & S-1 & 2 & 4 & 8 & 7 \\
\hline $64, \mathrm{M}$ & 2 & $\mathrm{~T}-7$ & $4-$ & $4+$ & 1 & 1 \\
\hline $74, \mathrm{M}$ & 3 & T-5 & 5 & 5 & 0 & 0 \\
\hline $48, \mathrm{M}$ & 12 & $\mathrm{~T}-6$ & $4+$ & $4+$ & 3 & 0 \\
\hline $69, \mathrm{M}$ & 36 & T-9 & 1 & 1 & 8 & 8 \\
\hline $60, \mathrm{M}$ & 1 & S-1 & 5 & 5 & 3 & 1 \\
\hline
\end{tabular}

that early treatment is associated with an improved likelihood of neurological recovery. Certainly, there is no reason to delay intervention once a dAVF has been identified and deemed amenable to treatment.

Contemporary data suggest that some spinal dAVFs are amenable to endovascular therapy. ${ }^{1,13}$ Such techniques are 
less invasive and allow for diagnosis and treatment in a single session in a subset of cases. Unfortunately, endovascular therapy has been associated with lower initial success rates and higher rates of recurrence compared with microsurgical treatment, ranging from $15 \%$ to $44 \%$. $^{6,7,15,18}$ Eskandar et al. reported a failure rate of endovascular therapy of $39 \%$, whereas the failure rate of surgical therapy was $0 \% .{ }^{5}$ Gokhale et al. found a $30 \%$ recurrence rate after endovascular therapy with one case of spinal infarction, ${ }^{6}$ and Ruiz-Juretschke et al. cited a $44 \%$ failure rate after endovascular treatment. ${ }^{14}$ Because these fistulas are often fed by multiple dural arterial vessels with a single draining vein, ${ }^{12}$ the inability to occlude all arterial feeders through an endovascular approach can lead to recanalization or development of collaterals. ${ }^{13}$ Embolization is also limited in cases with small arterial feeders, patients with severe atherosclerosis, or a feeding vessel originating from the same segmental artery as the artery of Adamkiewicz. ${ }^{13}$

Historically, surgical treatment is associated with high success rates. A meta-analysis of 16 surgical studies found successful occlusion of spinal dAVFs in $98 \%$ of cases with only $2 \%$ morbidity, compared with $46 \%$ occlusion and $4 \%$ morbidity in an endovascular treatment group. ${ }^{2,17}$ The same study found clinical improvement in 55\% and stabilization in $34 \%$ among surgically treated patients, while those who underwent endovascular therapy had initial occlusion in $46 \%$ of cases with a morbidity rate of less than $4 \%$. This comparison is limited by selection bias, but other single-center cohorts have also reported similar results. ${ }^{16}$ Steinmetz et al. found that surgical treatment had an $89 \%$ chance to at least obtain stabilization of the preoperative clinical condition. ${ }^{17}$

Spinal dAVFs are found throughout the spine, but most commonly in the middle and lower thoracic spine. There are data suggesting that those in the lower thoracic spine improve more than in other locations, ${ }^{3}$ presumably due to improved vascular supply to the lower spinal cord, but the reasons remain unclear. In our cohort we made similar observations with higher rates of improvement in MRC and ALS scores among lesions in the lower thoracic and lumbosacral levels compared with those in the upper thoracic spine, yet none of these reached statistical significance.

There are several limitations of the present study. The cohort is small and follow-up is limited, but this is largely due to the fact that most lesions are referred to our center because of complex anatomy and inability to be treated by endovascular techniques. Referral bias also increases the complexity of lesions in our cohort. This study reviewed patients treated over a 20-year span, but the microsurgical technique remained remarkably stable over this time. Surgical treatment is simple interruption of the fistula intradurally on the venous side as the vein exits the fistula. The advent of ICG video angiography has facilitated the localization of dAVFs, and the extent of surgical exposure has generally shrunk over time to just 1-level laminectomies.

\section{Conclusions}

Surgical obliteration is associated with excellent outcomes in patients with spinal dAVFs. In this series, $78 \%$ of symptomatic patients demonstrated some form of clinical neurological improvement with $100 \%$ obliteration and no worsening of neurological function at last follow-up. Increased awareness of this diagnosis, recognition of pathognomic findings on MRI, and prompt spinal angiography are needed to avoid delays in diagnosis. There is a trend toward improved outcomes in patients with shorter symptom duration, stressing the need for prompt diagnosis and intervention. There is also a trend toward increased neurological improvement for dAVFs in the lower thoracic and lumbosacral levels compared with those in the upper thoracic cord.

\section{References}

1. Andres RH, Barth A, Guzman R, Remonda L, El-Koussy M, Seiler RW, et al: Endovascular and surgical treatment of spinal dural arteriovenous fistulas. Neuroradiology 50:869876, 2008

2. Cecchi PC, Musumeci A, Faccioli F, Bricolo A: Surgical treatment of spinal dural arterio-venous fistulae: long-term results and analysis of prognostic factors. Acta Neurochir (Wien) 150:563-570, 2008

3. Cenzato M, Debernardi A, Stefini R, D’Aliberti G, Piparo M, Talamonti G, et al: Spinal dural arteriovenous fistulas: outcome and prognostic factors. Neurosurg Focus 32(5):E11, 2012

4. Cho WS, Kim KJ, Kwon OK, Kim CH, Kim J, Han MH, et al: Clinical features and treatment outcomes of the spinal arteriovenous fistulas and malformation: clinical article. J Neurosurg Spine 19:207-216, 2013

5. Eskandar EN, Borges LF, Budzik RF Jr, Putman CM, Ogilvy CS: Spinal dural arteriovenous fistulas: experience with endovascular and surgical therapy. J Neurosurg 96 (2 Suppl):162-167, 2002

6. Gokhale S, Khan SA, McDonagh DL, Britz G: Comparison of surgical and endovascular approach in management of spinal dural arteriovenous fistulas: a single center experience of 27 patients. Surg Neurol Int 5:7, 2014

7. Hall WA, Oldfield EH, Doppman JL: Recanalization of spinal arteriovenous malformations following embolization. J Neurosurg 70:714-720, 1989

8. Hassler W, Thron A, Grote EH: Hemodynamics of spinal dural arteriovenous fistulas. An intraoperative study. J Neurosurg 70:360-370, 1989

9. Hurst RW, Kenyon LC, Lavi E, Raps EC, Marcotte P: Spinal dural arteriovenous fistula: the pathology of venous hypertensive myelopathy. Neurology 45:1309-1313, 1995

10. Jellema K, Canta LR, Tijssen CC, van Rooij WJ, Koudstaal PJ, van Gijn J: Spinal dural arteriovenous fistulas: clinical features in 80 patients. J Neurol Neurosurg Psychiatry 74:1438-1440, 2003

11. Koenig E, Thron A, Schrader V, Dichgans J: Spinal arteriovenous malformations and fistulae: clinical, neuroradiological and neurophysiological findings. J Neurol 236:260-266, 1989

12. McCutcheon IE, Doppman JL, Oldfield EH: Microvascular anatomy of dural arteriovenous abnormalities of the spine: a microangiographic study. J Neurosurg 84:215-220, 1996

13. Park SB, Han MH, Jahng TA, Kwon BJ, Chung CK: Spinal dural arteriovenous fistulas: clinical experience with endovascular treatment as a primary therapeutic modality. $\mathbf{J}$ Korean Neurosurg Soc 44:364-369, 2008

14. Ruiz-Juretschke F, Perez-Calvo JM, Castro E, GarcíaLeal R, Mateo-Sierra O, Fortea F, et al: A single-center, long-term study of spinal dural arteriovenous fistulas with multidisciplinary treatment. J Clin Neurosci 18:1662-1666, 2011 
15. Song JK, Gobin YP, Duckwiler GR, Murayama Y, Frazee JG, Martin NA, et al: N-butyl 2-cyanoacrylate embolization of spinal dural arteriovenous fistulae. AJNR Am J Neuroradiol 22:40-47, 2001

16. Song JK, Vinuela F, Gobin YP, Duckwiler GR, Murayama Y, Kureshi I, et al: Surgical and endovascular treatment of spinal dural arteriovenous fistulas: long-term disability assessment and prognostic factors. J Neurosurg 94 (2 Suppl):199-204, 2001

17. Steinmetz MP, Chow MM, Krishnaney AA, AndrewsHinders D, Benzel EC, Masaryk TJ, et al: Outcome after the treatment of spinal dural arteriovenous fistulae: a contemporary single-institution series and meta-analysis. Neurosurgery 55:77-88, 2004

18. Van Dijk JM, TerBrugge KG, Willinsky RA, Farb RI, Wallace MC: Multidisciplinary management of spinal dural arteriovenous fistulas: clinical presentation and long-term follow-up in 49 patients. Stroke 33:1578-1583, 2002

\section{Disclosures}

Dr. Clark reports being a consultant to NuVasive.

\section{Author Contributions}

Conception and design: Lawton, Clark. Acquisition of data: Safaee, Clark. Analysis and interpretation of data: Lawton, Safaee, Clark, Burkhardt. Drafting the article: all authors. Critically revising the article: all authors. Reviewed submitted version of manuscript: all authors. Statistical analysis: Safaee, Clark. Administrative/technical/material support: Lawton. Study supervision: Lawton.

\section{Correspondence}

Michael T. Lawton: Barrow Neurological Institute, Phoenix, AZ. michael.lawton@barrowbrainandspine.com. 